\title{
Force-Velocity Relation: Its Implications about Molecular Mechanism of Muscle Contraction
}

\author{
Sugi $\mathrm{H}^{1 *}$ and Chaen $\mathbf{S}^{2}$ \\ ${ }^{1}$ Department of Physiology, School of Medicine, Teikyo University, Tokyo, Japan \\ ${ }^{2}$ Department of Biosciences, College of Humanities and Sciences, Nihon University, Tokyo, Japan
}

\begin{abstract}
In 1938, Hill described the relation between force $(P=l o a d)$ and initial velocity of shortening $(V)$ in contracting whole frog muscles as part of rectangular hyperbola: $(P+a) V=b(P o-P)$, where $P o$ is the maximum isometric force and $a, b$ are constants. This equation indicates that muscle can regulate its energy output depending to the amount of load imposed on it. In 1954, H.E. Huxley and Hanson made a monumental discovery that muscle contraction results from relative sliding between actin and myosin filaments, providing structural basis to study and discuss mechanisms underlying the P-V relation. In 1957, A.F. Huxley has constructed a contraction model, in which muscle contraction characteristics including the $\mathrm{P}-\mathrm{V}$ relation are explained in terms of attachment-detachment cycle between myosin heads extending from myosin filaments and the site in actin filaments. The Huxley contraction model stimulated the interest of muscle investigators to study the P-V relation using intact single muscle fibers with the following results: (1) the shape of P-V relation deviates from hyperbola at loads $>\sim 0.8 \mathrm{Po}$; (2) at the beginning of muscle shortening under lads $<\mathrm{Po}$, the fibers show non steady shortening before shortening with a constant velocity velocity; and (3) if loads >Po is imposed on muscle fibers, they show remarkable length oscillations before being stretched. To eliminate the gap between muscle contraction characteristics and biochemical studies on actomyosin ATPase reaction, experiments are now performed using skinned muscle fibers, from which surface membrane is removed. In this article, we not only describe contraction models other than the Huxley contraction model to explain the results obtained, but also experimental techniques to obtain P-V relations especially from skinned muscle fibers having structural instability.
\end{abstract}

Keywords: Muscle Contraction; Force-velocity relation; Huxley contraction model; Sliding filament mechanism; Skinned muscle fiber

\section{Historical Background}

Muscle contraction can be expressed in two different ways, in terms of shortening or in terms of force (=tension). Two corresponding methods are used for measuring activity of the contractile system in muscle. In one method, muscle shortening is recorded while the muscle is made to contract under a constant load (isotonic contraction or isotonic shortening). In the other method, muscle is fixed in position at both ends, and force development is recorded during contraction (isometric contraction). Under zero external load, muscle shortens with the maximum velocity $V_{\max }$. Muscle shortening velocity $(V)$ decreases with increasing external load (=force, $P$ ), reaching to zero when the load is increased to Po, i.e. the maximum isometric force generated by muscle. If muscle at its slack length $L o$ (i.e. the length at which resting tension is negligible) is held in the experimental device shown in Figure 1 , and stimulated maximally, it first generates isometric force $P$ until it reaches to a value equal to the external load, and then starts shortening with a constant velocity $V$.

In 1938, Hill studied the relation between $P$ and $V$ using whole frog skeletal muscle, and reported that the shape of the $P-V$ relation was a rectangular hyperbola, so that

$$
\begin{aligned}
& (P+a)(V+B)=\text { constant }=(P o+a) b, \text { or, } \\
& (P+a) V=b(P o-P)
\end{aligned}
$$

Where $a$ and $b$ are constants (Figure 2) [1]. Hill further claimed that the constant $a$ was equal to the heat constant $a$, i.e., the amount of heat liberated per unit distance of muscle shortening, though the two constant were later proved not to be the same. If, however, $P V$ and $a V$ are approximately regarded as work flux and heat flux from contracting muscle, respectively, the above equation implies that the muscle can regulate its rate of energy (heat + work) liberation depending on the amount of load imposed on it [2]. The curvature of hyperbolic $P-V$ relation is determined by the value of $a / P o$, which is generally taken as a measure of efficiency, with which muscle converts chemical energy derived from ATP hydrolysis into mechanical work. In frog sartorius muscle at $0^{\circ} \mathrm{C}, \mathrm{a} / \mathrm{Po}$ is 0.25 , while the maximum velocity of muscle shortening under zero load is $\sim 1.6 \mathrm{Lo} / \mathrm{s}$, and Po is $\sim 2 \mathrm{~kg} / \mathrm{cm}^{2}[1]$.

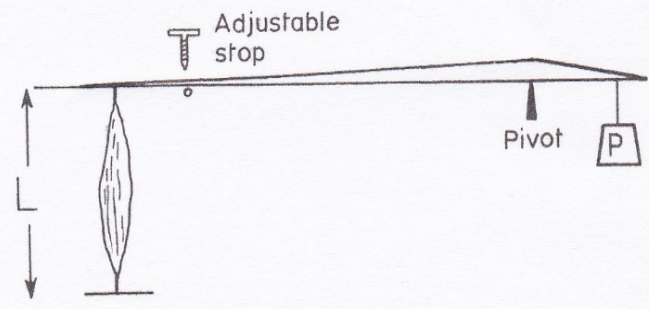

Figure 1: A mechanical device to record length and force changes of muscle during isotonic shortening. Muscle length is first adjusted to Lo with an adjustable stop. When muscle is stimulated maximally, a stop (small circle under the lever) is quickly removed so that muscle shortens by lifting a load $P$. Muscle shortening is recorded with a photoelectric device, while muscle force is recorded with a force transducer (not shown).

*Corresponding author: Sugi $\mathrm{H}$, Department of Physiology, School of Medicine Teikyo University, Tokyo, Japan, Tel: +81484784079; E-mail: sugi@kyf.biglobe.ne.jp

Received September 28, 2016; Accepted October 06, 2016; Published October 13,2016

Citation: Sugi H, Chaen S (2016) Force-Velocity Relation: Its Implications about Molecular Mechanism of Muscle Contraction. J Nanomed Nanotechnol 7: 401. doi: 10.4172/2157-7439.1000401

Copyright: ( 2016 Sugi $\mathrm{H}$, et al. This is an open-access article distributed unde the terms of the Creative Commons Attribution License, which permits unrestricted use, distribution, and reproduction in any medium, provided the original author and source are credited. 


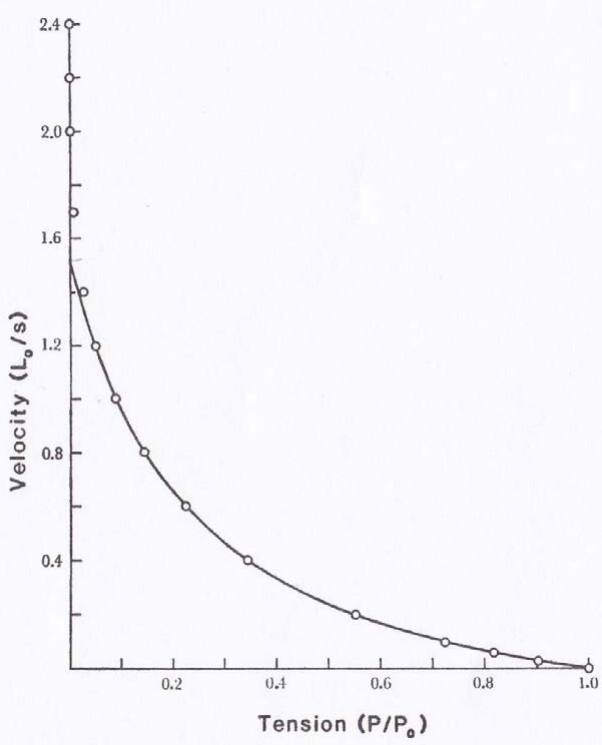

Figure 2: Force-Velocity relation of whole frog Sartorius muscle. The curve fits part of rectangular hyperbola, so that the relation between shortening velocity $V$ and force $P$ is expressed as, $(a+P) V=b(P o-P)$.

The mechanism underlying the force-velocity $(P-V)$ relation was a mystery at that time, because of complete lack of knowledge about muscle structures. However, the monumental discovery of H.E. Huxley and Hanson in 1954 [3] that muscle contraction results from relative sliding between actin and myosin filaments provided structural basis to explain and discuss muscle contraction characteristics including the $\mathrm{P}-\mathrm{V}$ relation in the light of sliding filament mechanism. In this article, we intend to discuss mechanisms underlying the P-V relation, which we think most interesting and important, but not intend to make an extensive survey of the papers published in the past.

\section{A.F. Huxley's 1957 Contraction Model}

Based on the discovery of sliding filament mechanism by H.E. Huxley and Hanson, a number of contraction models have hitherto been presented. Among them, the contraction model of A.F. Huxley has been central in the research field of muscle contraction mechanism [4]. The main assumptions, which have been made to explain Hill's experimental results, can be summarized as follows.

- During muscle contraction, myosin heads (cross-bridges) have only two states, i.e. attached (to actin) and detached (from actin) states.

- As shown in Figure 3A, a myosin head (M) connected to thick (myosin) filament with springs $\mathrm{S}_{1}$ and $\mathrm{S}_{2}$ fluctuates around its equilibrium position $\mathrm{O}$, due to thermal agitation. If it attaches to an active site on thin (actin) filament, it exerts either positive or negative force $F$ proportional to its distance $x$ by pulling $\mathrm{S}_{1}$ or $\mathrm{S}_{2} . F=0$ when $x=0, F=\mathrm{k} x$ when $x>0$ and $F=-\mathrm{k} x$ when $x<0, \mathrm{k}$ is the stiffness of spring.

- Rate constants for attachment of $\mathrm{M}$ and its detachment from, actin filament ( $f$ and $g$, respectively) are functions of $x$ as shown in Figure $3 \mathrm{~B}$. $\mathrm{M}$ attaches to $\mathrm{A}$ if $\mathrm{M}$ and $\mathrm{A}$ are within the range of $x$ where $f$ has finite values $(0 \leq x \leq h)$ to exert positive for the filament sliding by pulling $\mathrm{S}_{1}$. If the A-M link moves across 0 to the negative $x$ region, it beaks fairly rapidly because $f$ is zero and $g$ has a large value.
In Figure 3C, distribution of A-M links (expressed by fractional numbers $0 \leq n \leq 1)$ is shown against $x$ at various constant velocities $V$ (expressed relative to $V_{\max }$ ). In the isometric condition, in which $P=P o$, and no filament sliding takes place, A-M links are distributed only in the positive $x$ region where $f$ has finite values $(0 \leq x \leq h)$. As the constant shortening velocity increases, the number of A-M links exerting positive forces decreases as a result of moderate values of $f$; the proportion of $\mathrm{M}$ that slide past $\mathrm{A}$ without formation of $\mathrm{A}-\mathrm{M}$ link increases with increasing velocity with which myofilaments slide past each other. Meanwhile, A-M links brought into the negative $x$ region tend to exist over larger distance as the sliding velocity between the filaments increases. Finally, the sliding velocity reaches a value, under which the positive and the negative forces by A-M links are equal. This value corresponds to the maximum shortening velocity $V_{\max }$ under zero load.

In this contraction model described above, values of $f$ and $g$ in the positive $x$ region were chosen to be consistent with Hill's heat measurement data in frog muscle during isometric contraction (maintenance heat), while the value of $g$ in the negative $x$ region was determined to fit the $P-V$ relation in frog skeletal muscle [1].

\section{Force-velocity Relation Obtained from Single Intact Muscle Fibers}

Since the whole muscle consists of a number of muscle fibers with variable contraction characteristics and includes blood vessels and

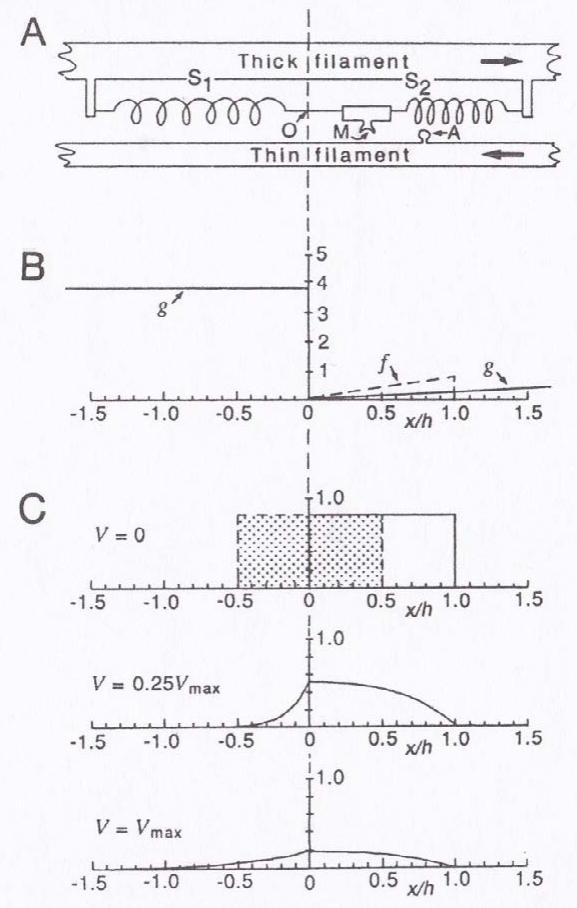

Figure 3: Huxley 1957 contraction model which explains the P-V relation in terms of distribution of myosin head attached to actin filament. (A) Diagram showing a myosin head (M) connected to thick (myosin) filament with springs $S_{1}$ and $S_{2}$, and the active site $(A)$ of thin (actin) filament. Arrows indicate directions of relative sliding between the two filaments. (B) Dependence of $f$ and $g$ on the distance $(x)$ of $M$ from its equilibrium position (0) to explain the hyperbolic P-V relation shown in Figure 2. (C) Diagrams showing distribution of A-M links with respect to $x$ at $V=0$ i.e. isometric condition (upper diagram), at $\mathrm{V}=0.25 \mathrm{Vmax}$ (middle diagram), and $\mathrm{V}=\mathrm{V} \max$ (lower diagram). Broken line and shaded area show distribution of $A^{\wedge} M$ ink when muscle length is suddenly changed to drop Po to zero. 
connective tissues, more precise experiments using isolated single muscle fibers are desired. Isolation of intact single fibers from muscle, however, requires enormous technical skill, and only limited research groups can study contraction characteristics of single muscle fibers. Normally, single fibers isolated from frog semitendinosus or tibialis anterior muscles are used.

Edman [5] obtained P-V relation in isotonically shortening from single intact frog muscle fibers by the method essentially the same as that used by Hill, and found that the $P-V$ relation was double hyperbolic in shape, and markedly deviated from the hyperbola at loads $\geq 0.8 P o$ (Figure 4), so that a distinct hump appeared in the P-V curve in the high force region. This indicates that the kinetics of attachment-detachment cycle between myosin heads and actin filaments is different between the low force and the high force regions. The double hyperbolic shape of $\mathrm{P}-\mathrm{V}$ relation in single intact frog muscle fibers by Iwamoto et al. [6]. As will be described later, analogous $P-V$ relations with distinct hump in the high force region has also been obtained from skinned rabbit psoas muscle fibers, from which surface membrane is removed, indicating that the deviation of $\mathrm{P}-\mathrm{V}$ relation from the hyperbola at high force region is a fundamental characteristic of the contractile system common to amphibian and mammalian muscle fibers.

Cecchi et al. [7] obtained P-V relation of single frog muscle fibers during the development of isometric force. Using an electronic servo motor system, they applied a ramp-shaped constant-velocity (ramp) decrease in fiber length to a maximally stimulated muscle fiber at various times after the beginning of isometric force development as illustrated in Figure 5A, with or without initial small step decrease in fiber length (solid and broken line traces, respectively) with qualitatively similar results; during each release with a constant velocity $V$, the force first fell from $P o$ to a lower value $P<P o$, which was kept constant during the course of release. Thus, they obtained $P-V$ relations at various times during the course of isometric force development as shown in Figure 5B. The value of $V_{\max }$ was found to remain unchanged irrespective of the time during the development of isometric tension. This result can easily be accounted for on the basis of the Huxley contraction model, since $V_{\max }$ results from the balance between positive and negative forces at both sides of the equilibrium position 0 , and therefore independent of the number of A-M links (Figure 3C). Meanwhile, Cecchi et al. did not

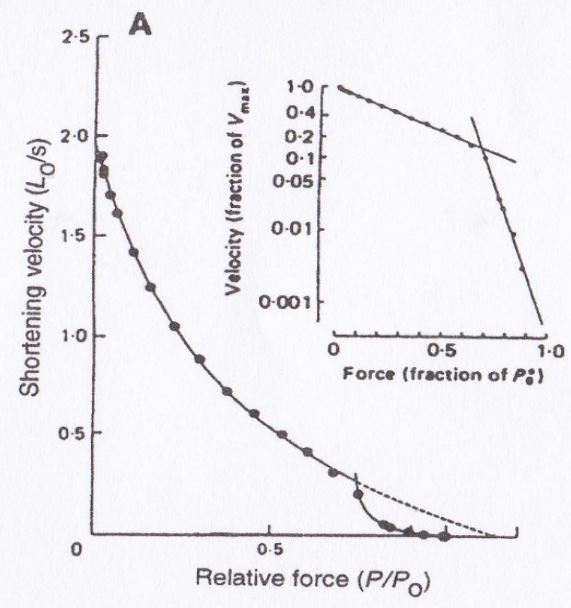

Figure 4: Double hyperbolic $P-V$ relation obtained from single intact frog muscle fibers. Inset shows semi logarithmic plot of the same $P-V$ data. From Edman [5].
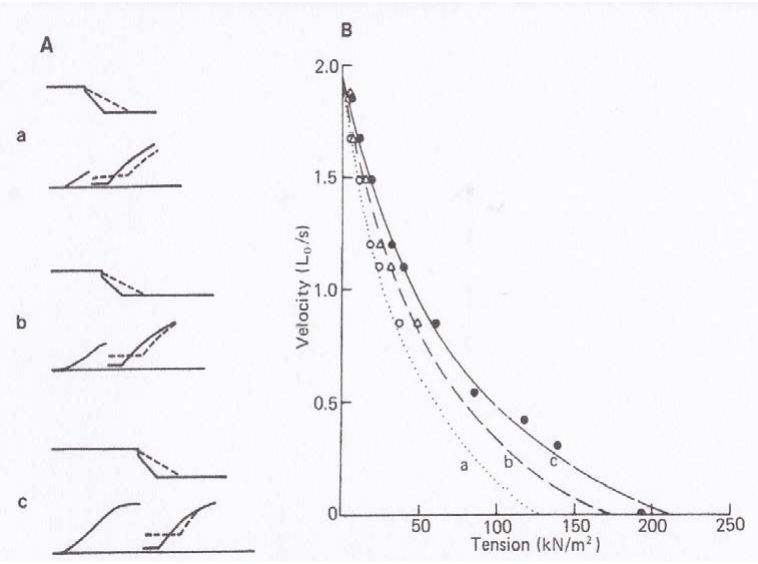

Figure 5: $P-V$ relations obtained at various times during the course of isometric force development. (A) Records showing length (upper traces) and force (lower traces) changes when the fiber was subjected to ramp-shaped releases with (solid line) or without (broken line) at $75 \mathrm{~ms}$ (a), $100 \mathrm{~ms}$ (b), and $190 \mathrm{~ms}$ (c) after the beginning of stimulus. (B) $P-V$ relations obtained at $75 \mathrm{~ms}(\mathrm{a}), 100 \mathrm{~ms}$ (b), and $190 \mathrm{~ms}$ (c) after the beginning of stimulus. From ref. [6].

observe the double hyperbolic shape of $P$ - $V$ relation, probably because they did not obtain enough data points in the high force region.

Iwamoto et al. [6] made interesting experiments, in which single frog muscle fibers are made to shorten against an auxotonic load, i.e. a spring whose force increases linearly with the extent of lengthening. They obtained $P-V$ relations under various auxotonic loads (Figure 6). The shape of the auxotonic $P-V$ relation was convex upwards, reflecting the extremely slow velocity when the fiber starts shortening under auxotonic loads. They further showed that, if the values of $V$ are plotted against auxotonic force $P$ normalized relative to the isometric force Piso, which is reached at the same time $t$ after the beginning of auxotonic shortening (Figure 7A), the P/Piso versus $V$ relation fitted fairly well to the isotonic $\mathrm{P}-\mathrm{V}$ relation in the high force region (Figure 7B). This seems to suggest that the kinetics of actin-myosin interaction might be more or less similar during both isotonic shortening and auxotonic shortening in the high force region.

\section{Isotonic Velocity Transients and Its Explanation in Terms of Attached Myosin Head Distribution}

Experiments with whole frog muscle are inadequate to record time course of muscle shortening at its early phase, because of mechanical vibrations when muscle starts shortening against a massive load [8]. To make this point clear, Civan and Podolsky [9] performed experiments, in which the early phase of isotonic shortening of isolated single frog muscle fibers was recorded. To avoid mechanical vibrations at the beginning of shortening, they used a steel spring with a length much longer than the distance of fiber shortening, so that the fibers shortened by pulling the long spring, so that the fiber shortening took place under practically constant load. By this method, they could successfully record the early phase of isotonic fiber shortening. They found that, at the beginning of isotonic shortening, the fiber first showed non steady shortening resembling damped sine waves. As can be seen in Figure 8 , the fiber shortens initially at a velocity higher than the subsequent constant velocity. The velocity then slows down and again increase until it reaches a constant value. They called this non steady period of fiber shortening as "isotonic velocity transients".

As the Huxley contraction model (Figure 3) only describes distribution of myosin head attached to actin filament during constant 


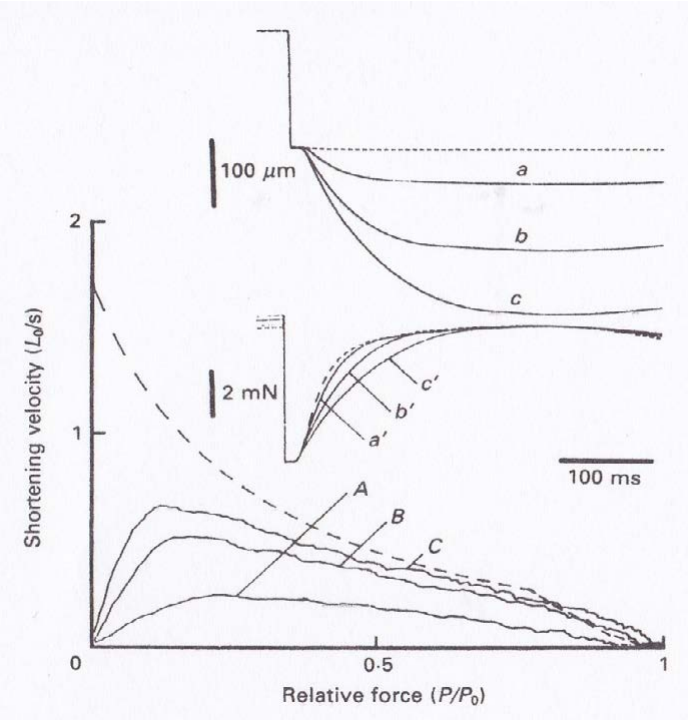

Figure 6: $P-V$ relations under various auxotonic loads in maximally stimulated single frog muscle fibers. Broken line indicates the double hyperbolic P-V relation obtained from isotonic shortening experiments. Inset shows length (upper traces) and force (lower traces) changes when a muscle fiber generating the maximum isometric force Po is first made slack, and then subjected to auxotonic loads with different compliance values. Dotted lines in both length and force records show the development of isometric force when the fibe length is kept at the slackened length. Note that, after being made to slack, the fiber starts shortening against auxotonic load with extremely small velocities compared to the velocity of subsequent shortening. From ref [7].
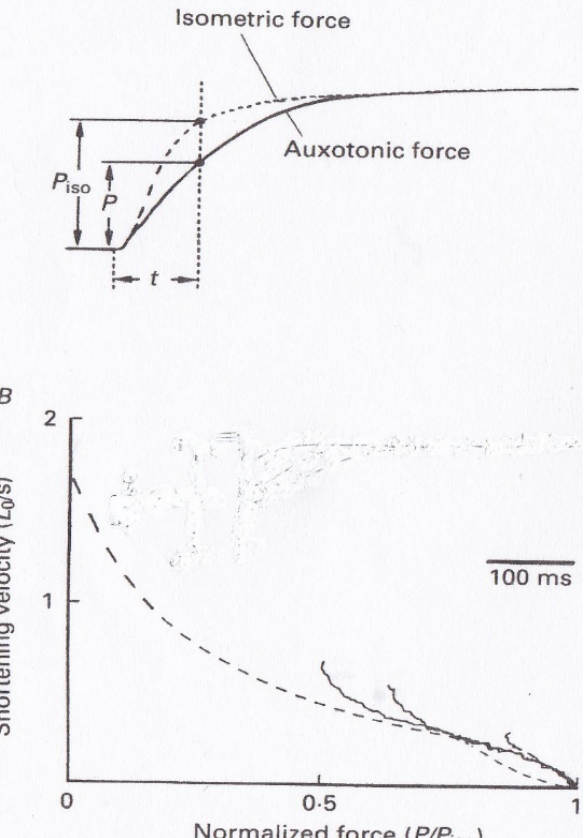

Figure 7: $P-V$ relation during auxotonic shortening after normalization of forces $P$ during auxotonic shortening. (A) Diagram showing method of normalization of $P$ relative to $P$ iso during isomeric force development at the same time $t$ after the beginning of auxotonic shortening and force development. (B) The P/Piso versus $V$ relations under different auxotonic loads (solid lines) fits fairly well to the $P-V$ relation (broken line) during isotonic shortening in the high force region. From ref. [7]. velocity shortening, Podolsky and Nolan [10] proposed another contraction model to account for the isotonic velocity transients (Figure 9). In contrast with the Huxley contraction model, the Podolsky-Nolan model assumes large values of $f$ and a very small value of $g$ in the positive $x$ region, so that all myosin heads passing through this region form A-M links irrespective the velocity of filament sliding. In the negative $x$ region, $g$ remains to be very small over a distance from the equilibrium position 0 and then increases to a large value (Figure 9). As the result, the mode of distribution of A-M link under various loads is markedly different from that in the Huxley contraction model (Figure 10). By some additional assumptions, the Podolsky-Nolan model can explain not only the isotonic velocity transients, but also other muscle contraction characteristics and also heat measurement results.

\section{Marked Oscillation of Fiber Length Following Step Increases in Load $>$ Po}

Sugi and Tsuchiya [11] studied both the isotonic shortening and the isotonic lengthening of frog muscle fibers under loads smaller or

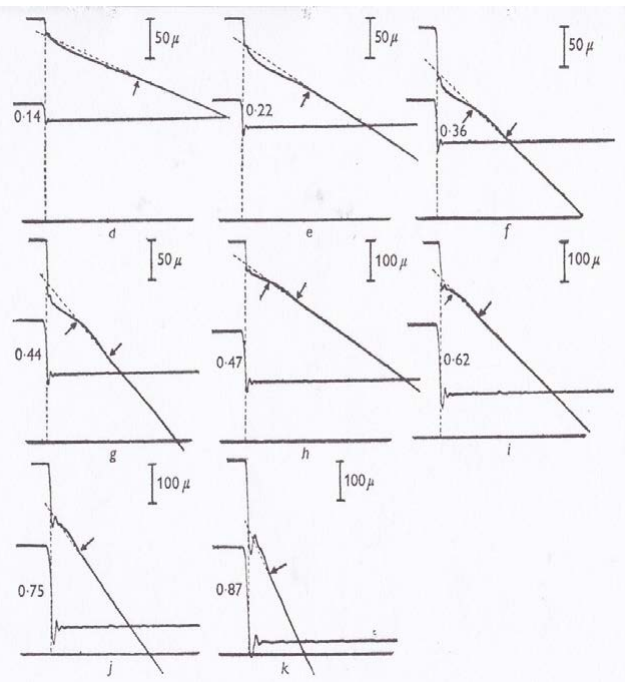

Figure 8: Isotonic velocity transients in single frog muscle fibers following various magnitudes of quick changes in load from $P_{0}$ to $P<P o$. Upper and lower records show fiber shortening and step changes in load, respectively. The magnitude of step changes in load is given as fractions of $P$ o. From ref. [9]

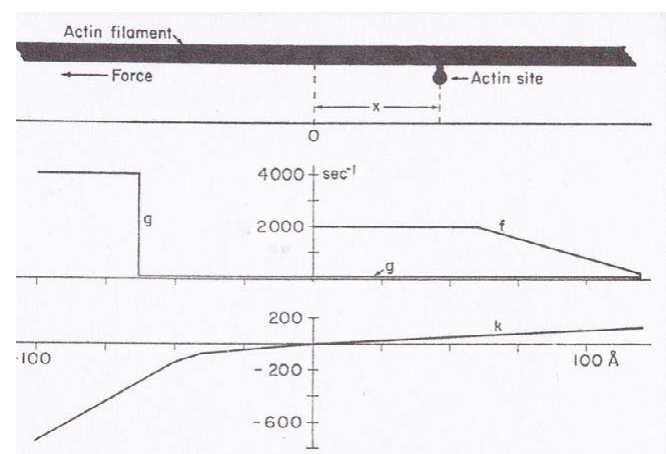

Figure 9: Podolsky-Nolan contraction model constructed to explain the isotonic velocity transient. Upper and middle panel show $f$ and $g$, i.e. rate constants for formation and breaking of A-M link as function of distance between a myosinbinding site on actin filament and the position 0 at which A-M link exerts no force. Lower panel shows dependence of elastic constant $k$ of A-M link on $x$. 
larger than the maximum isometric force Po by the method shown in Figure 11.

Though the method was essentially the same as that used by Civan and Podolsky, they combined three loading spring and to levers to apply various loads $(P \leq \mathrm{Po}$ or $\mathrm{P} \geq \mathrm{Po}$ ) to the fibers not only in one step but also in two arbitrary steps. Figure 12 shows typical results of experiments, in which the load on the isometrically contracting fibers was suddenly changed from $P o$ to $P<P o$ (left) or from $P$ o to $P>P$ o (right). The early time course of isotonic shortening was similar to the isotonic velocity transients reported by Civan and Podolsky [9], while the early time course of isotonic lengthening was variable. For the loads $P<1.31 P o$, the fibers were lengthened with velocities increasing with time. For the loads $P<1.20 \mathrm{Po}$, on the other hand, the fibers exhibited oscillatory length changes with reversal in the direction of movement before starting to lengthen continuously. Two load step experiments showed that the oscillatory length changes with alternate lengthening

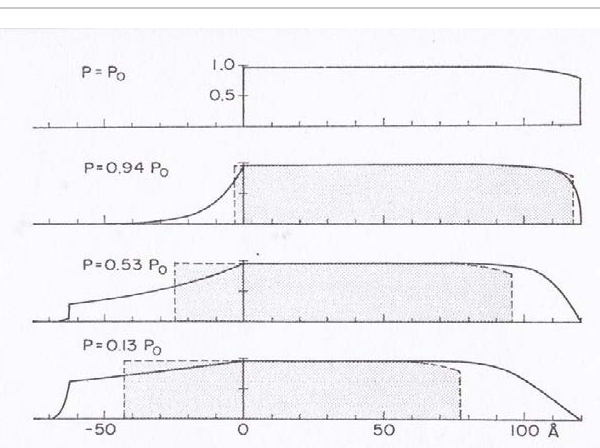

Figure 10: Diagrams showing distribution of A-M links with respect to distance $x$ from 0 during isotonic shortening following quick changes in load from $P o$ to $P<P$. Values of $P$ are given at the left of diagrams. In each diagram, A-M link distribution immediately after quick changes in load is given by shaded area, while the subsequent steady A-M link distribution is given by solid line. From ref. [10].

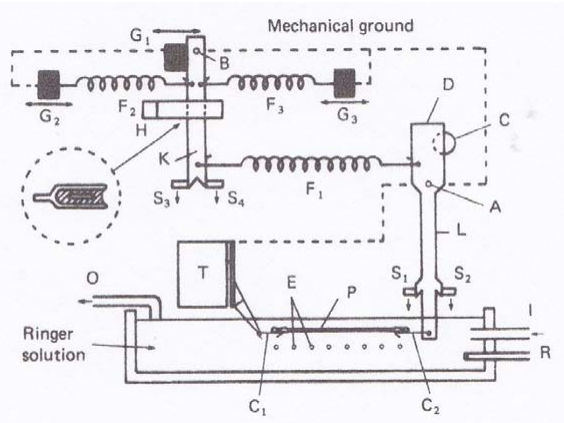

Figure 11: Schematic drawing of experimental arrangement to apply quick changes in load in two arbitrary steps. A single fiber $\mathrm{P}$ is mounted between force transducer $T$ and lever $L$ with clips $C_{1}$ and $C_{2}$, and stimulated maximally with $\mathrm{Pt}$ wire electrodes. Lever $\mathrm{L}$ is pivoted at $\mathrm{A}$ and loaded by spring $\mathrm{F}_{1}$, which is hooked to lever $L$ and another lever $K$ so that the length of $F_{1}$ can be changed by micromanipulator $G_{1}$ carrying $K$. Long arms of $L$ and $K$ are restrained by pairs of electromagnetically controlled stops $S_{1}$ and $S_{2}$ and $S_{3}$ and $S_{4}$, respectively. Short arm of $L$ serves as a vane interrupting light bean $C$ directed towards photodiode (not shown) to serve as displacement transducer recording fiber length changes. With a pair of additional springs $F_{2}$ and $F_{3}$, whose lengths are adjusted by microman ipulators $G_{2}$ and $G_{3}$, the length of $F_{1}$ can be changed quickly when $S_{3}$ and $S_{4}$ are removed to produce movement of K. Oscillation of $\mathrm{K}$ is damped with $\mathrm{Y}$ shaped dashpot device $\mathrm{H}$. After the fiber develops the maximum isometric force Po, stops restraining lever motion are removed in various sequences, so that the amount of load on the fiber can be changed in two arbitrary steps, as shown in Figure 12 and 13 From ref. [11].
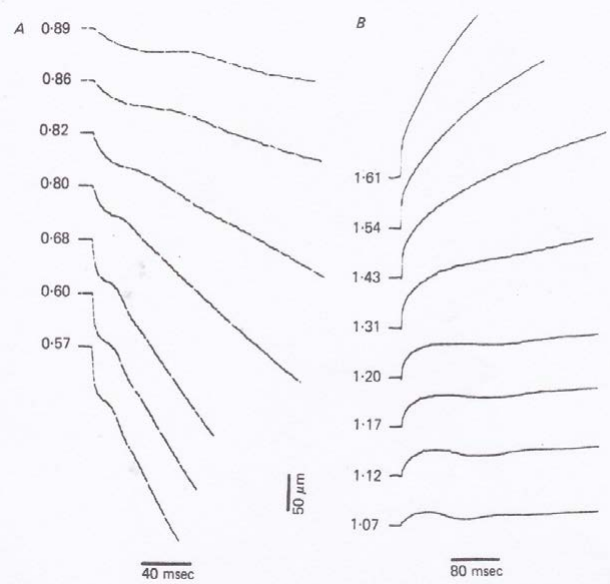

Figure 12: Isotonic velocity transients following quick changes in load from Po to $\mathrm{P}<\mathrm{Po}(\mathrm{A})$, and from $\mathrm{Po}$ to $\mathrm{P}>\mathrm{Po}(\mathrm{B})$. The values of $P$ are expressed relative to $P o$ on the left of each record. From ref. [11]

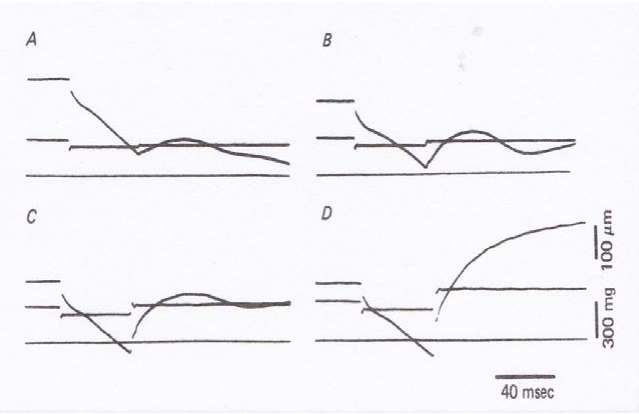

Figure 13: Records of experiments, in which the load on the fiber was increased quickly after a period of isotonic shortening under a load of $0.8 P o$. Note marked oscillatory length changes with alternate lengthening and shortening. From ref. [11].

and shortening were most marked when the load on the fibers was first reduced from $P_{0}$ to $\sim 0.8 P_{0}$, and then increased slightly after a period of isotonic shortening (Figure 13).

To account for the marked fiber length oscillations with alternate fibre lengthening and shortening shown in Figure 13, Sugi and Tsuchiya [11] constructed a contraction model shown in Figure 14. The dependence of the values of $f$ and $g$, i.e. the rate constant for the formation and the breaking of A-M links, on the distance $x$ from the equilibrium position 0 is analogous to that of the Podolsky-Nolan model [10] except that $g$ is infinitely large for $x>185 \AA$ and $x<185 \AA$. To explain the marked oscillatory changes in fiber length, an additional assumption is made that $g$ increases instantaneously in response to step increases in load from $P o$ to $P>P o$, and returns to the original value exponentially with time, so that the time course of change in $g$ following a step increase in load is expressed as $\mathrm{g}=\mathrm{g}_{0}+\mathrm{Ae}^{-\mathrm{Bt}}$, where $\mathrm{A}$ and $\mathrm{B}$ are constants determining the magnitude of instantaneous increase in $g$ and the time constant of subsequent exponential recovery of $g$, respectively.

As described above, no definite conclusion can be reached about what is actually taking place in muscle, though various contraction models have been presented to explain mechanical responses of muscle fibers in terms of changes in the A-M link distribution. Much more 
experimental work is needed for the full understanding of contraction mechanism.

\section{Force-Velocity Relation in Single Skinned Muscle Fibers}

To obtain information about the molecular mechanism of muscle contraction, the use of intact muscle fibers has serious limitations resulting from difficulties in precisely control chemical and ionic conditions around the myofilaments. The difficulties can be overcome by the use of skinned muscle fibers, from with surface membrane is removed by mechanical or chemical means. Since the contractile activity of muscle fibers are controlled by the release of $\mathrm{Ca}^{2+}$ from, and its uptake by the sarcoplasmic reticulum, skinned fibers can be fully activated with contracting solution $(\mathrm{pCa}, \sim 4)$, and can be relaxed with relaxing solution $(\mathrm{pCa},>9)$. To eliminate the gap between contraction characteristic of intact muscle or muscle fibers and biochemical studies on actomyosin ATPase reaction steps in solution, where the three-dimensional myofilament lattice is destroyed, skinned fibers are widely used and their characteristics including the force-pCa relation and MgATP concentration dependence of force development and shortening velocity have been obtained $[12,13]$. Another great advantage in the use of skinned fibers is that the rate of ATP hydrolysis by the contractile system can be measured simultaneously with mechanical experiments by measuring the rate of ADP production by NADH fluorescence [14].

Due to structural instability of skinned fibers, however, it is difficult to obtain enough data points to obtain $\mathrm{P}-\mathrm{V}$ relations, since fiber deterioration slowly proceeds in each contraction-relaxation cycle by the application of contracting and relaxing solutions [12]. We could overcome this difficulty by applying a ramp decrease in force from Po to zero to a maximally $\mathrm{Ca}^{2+}$-activated skinned fiber with an electronic device combined with a servo motor [15-17]. As can be seen in Figure $14 \mathrm{~A}$ that, in response to a ramp decrease in force applied at the plateau of $\mathrm{Ca}^{2+}$-activated maximum force $(\mathrm{Po})$, the fiber starts shortening with velocities increasing with linearly decreasing force, reaching $V_{\text {max }}$ when the force reaches zero force baseline. From the length and the force records, we can construct $\mathrm{P}-\mathrm{V}$ relation over the whole range of forces (=loads) imposed on the fiber, as will be described later.

Until the early 1980s, it was necessary for us to use hand-made mechanical or electronic elements to construct such an experimental setup such as shown in Figure 9, with enormous technical skill and patience, because the performance of commercially available electronic devices at that time were not satisfactory for our purpose. Fortunately, it is now possible to perform sophisticated mechanical experiments including multi-step load changes and ramp decreases in force with instruments commercially available (Aurora Scientific Inc., Aurora, Ontario, Canada), and we are currently performing research work with such electronic devices without using hand-made mechanical devices.

\section{P-V Relations are Useful in Specifying the Effect of Factors on the Contractile Mechanism in Muscle fibers}

Sugi et al. [16] examined the effect of polyclonal antibody to myosin subfragment-2, connecting myosin heads to myosin filament backbone, on skinned rabbit psoas muscle fibers by recording development of $\mathrm{Ca}^{2+}$-activated isometric force and MgATPase activity simultaneously. They found that, in the presence of the antibody ( 1.5 $\mathrm{mg} / \mathrm{ml}), \mathrm{Ca}^{2+}$-activated force development decreased with time and finally disappeared completely, while the MgATPase activity of the fibers remained unchanged (Figure 15). We also recorded P-V relation

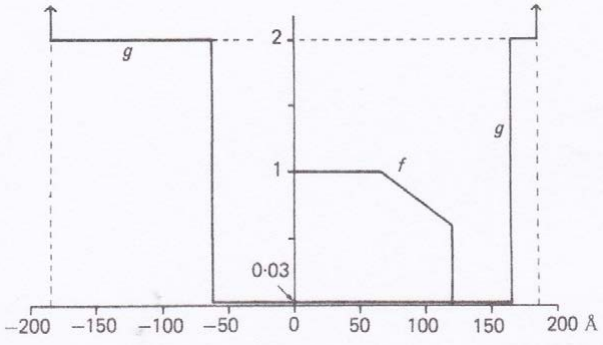

Figure 14: Sugi-Tsuchiya contraction model constructed to explain the marked oscillatory fiber length changes following quick increases in load. The values of rate constants $f$ and $g$ for the formation and the breaking of A-M links, respectively, are shown as functions of distance from the myosin head equilibrium position 0 . The value of $g$ is assumed to increase instantaneously in response to quick increases in load, and return to the initial value with a time course, $g=g_{0}+A e^{-B t}$, where $A$ and $B$ are constants. From ref. [11]

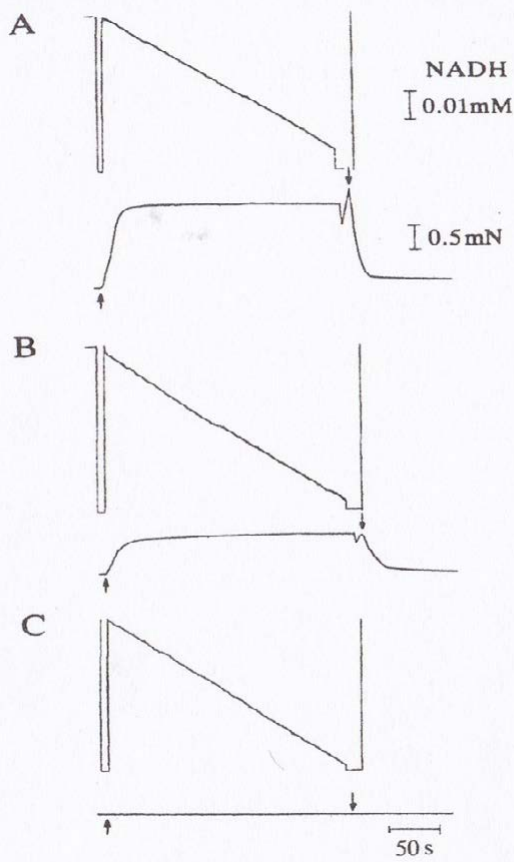

Figure 15: Effect of antibody to myosin subfragment-2 on the isometric force development and the MgATPase activity in skinned muscle fibers. Simultaneous recording of MgATPase activity (upper traces) and $\mathrm{Ca}^{2+}$-activated isomeric force development (lower traces) in skinned rabbit psoas muscle fibers. The records were obtained before application $(A)$, and after $100 \mathrm{~min}(B)$ and 150 min (C) after application of antibody to myosin subfragment-2 $(1.5 \mathrm{mg} / \mathrm{ml})$. Note that the slope of ATPase records does not change appreciably, while $\mathrm{Ca}^{2+}$-activated isometric force decreases with time and finally eliminated in the presence of antibody. Times of application of contracting and relaxing solutions are indicated by upward and downward arrows, respectively. From ref. [16].

before and at various times after application of the antibody, by applying ramp decreases in force at the plateau of $\mathrm{Ca}^{2+}$-activated isometric force (Figure 16). In response to ramp decreases in force, skinned fibers shortened with velocities increasing with decreasing force, reaching a maximum at zero force. The $P-V$ relations thus obtained are presented in Figure 16. Despite the decreased steady isometric force, the value of $V_{\max }$ remained unchanged (Figure 16A), and when the $P-V$ curves were found to be identical if they were normalized with respect to the maximum steady force attained (Figure 16B). These results indicate that the decrease in isomeric force by the antibody results from 
decrease in the number of myosin heads involved in force generation, while all myosin heads hydrolyze ATP irrespective of whether they generate force or do not generate force; in other words, individual myosin molecules in myosin filaments no longer generate force if the antibody attach to their subfragment- 2 region, while their ATPase activity remains unchanged (Figures 17 and 18).

On the other hand, it has been known that the magnitude of maximum isometric force $P o$ in skinned frog muscle fibers increases markedly at low ionic strength $[17,18]$, though the results were somewhat complicated due to gradual deterioration of the fibers. We examined the effect of low ionic strength on contraction characteristics and MgATPase activity of skinned rabbit psoas muscle fibers [19]. By gradually reducing ionic strength $\mu$ from standard value of $170 \mathrm{mM}$ up to $50 \mathrm{mM}$ by reducing $\mathrm{KCl}$ concentration in the experimental solution, both muscle fiber stiffness (measured by applying small sinusoidal vibration) and $\mathrm{Ca}^{2+}$-activated force increased with decreasing ionic strength;

The force at $0 \mathrm{mM} \mathrm{KCl}$ ( ionic strength, $50 \mathrm{mM}$ ) was $\sim$ two times larger than that at $125 \mathrm{M} \mathrm{KCl}$ (standard ionic strength, $170 \mathrm{mM}$ ) 0 $\mathrm{m}$ (Figure 19). We obtained P-V relations at various ionic strengths, and found that the value of $V_{\max }$ remained the same irrespective of the magnitude of maximum isometric force, which increased with
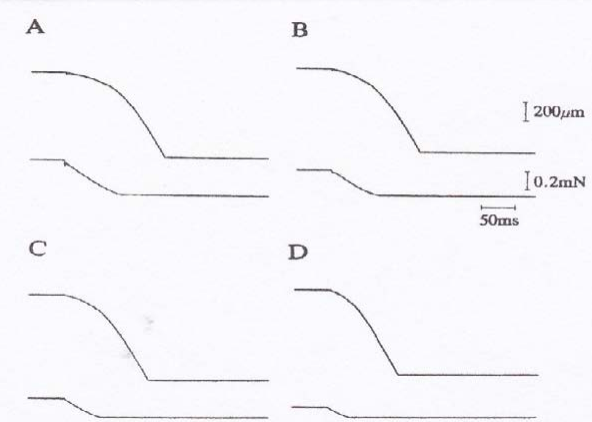

D

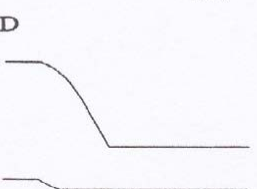

Figure 16: Shortening of $\mathrm{Ca}^{2+}$-activated skinned fibers in response to ramp decreases in force. Upper and lower records show fiber length and force changes in the fiber, respectively. Records A were taken before, and records B, $C$ and D were taken at 30,60 and $90 \mathrm{~min}$ after application of the antibody 1.5 $\mathrm{mg} / \mathrm{ml}$ ), respectively. From ref. [16].
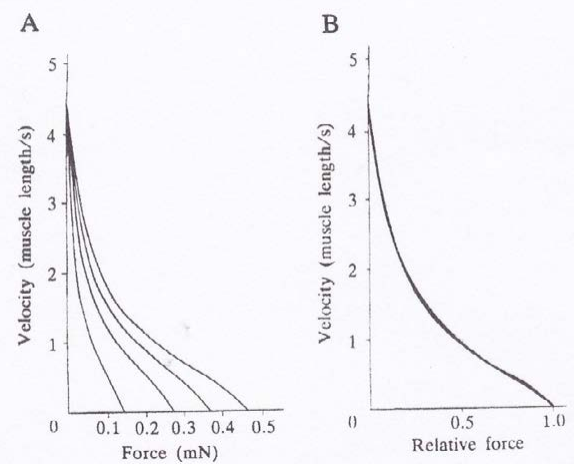

Figure 17: Effect of antibody to myosin subfragment-2 on the $P-V$ relation of $\mathrm{Ca}^{2+}$-activated skinned muscle fibers. (A) $P-V$ relations obtained before and at 30,60 and $90 \mathrm{~min}$ after application of the antibody. Note the value of $\mathrm{Vmax}$ remains unchanged despite decrease in the maximum force attained. (B) $P-V$ relations normalized with respect to the maximum force attained. Note that the curves are identical. From ref. [16].

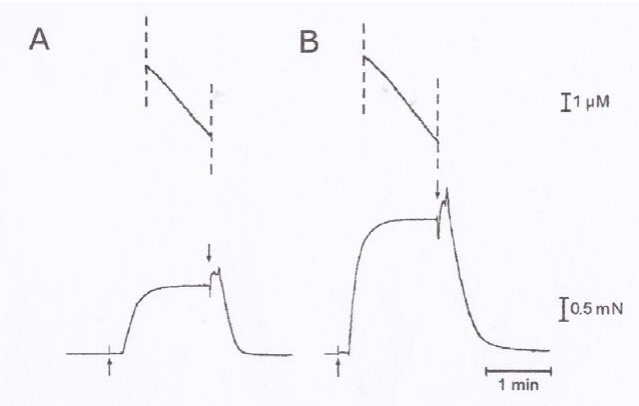

Figure 18: Effect of low ionic strength on MgATPase activity and $\mathrm{Ca}^{2+}$ activated isometric force development in skinned muscle fibers. MgATPase activity (upper records) and isometric force (lower records) at standard ionic strength $(170 \mathrm{mM})(A)$ and low ionic strength $(50 \mathrm{mM})(B)$. Note that the slope of MgATPase records does not differ appreciably between records in A and B, while the magnitude of isometric force increases $\sim$ twofold at low ionic strength. From ref. [19]
A

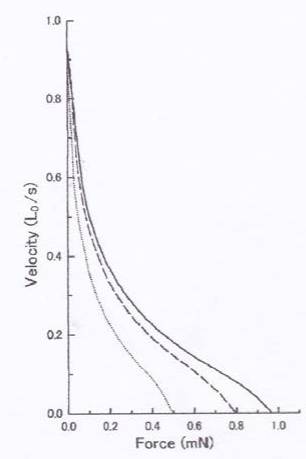

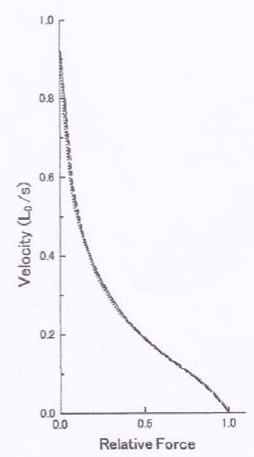

Figure 19: Effect of low ionic strength on the $P-V$ relation of $\mathrm{Ca}^{2+}$-activated skinned muscle fibers. (A) $P-V$ relations obtained at $0 \mathrm{mM} \mathrm{KCl}$ (solid line), at $50 \mathrm{mM} \mathrm{KCl}$ (broken line) and at $125 \mathrm{mM} \mathrm{KCl}$ (dotted line). Not that the value of $V$ max does not change appreciably despite decrease of steady isometric force with increasing $\mathrm{KCl}$ concentration. (B) The $\mathrm{P}-\mathrm{V}$ relations normalized with respect to the steady forces attained. Not that the P-V curves are identical in shape. From ref. [20].

decreasing ionic strength, and if the P-V curves are normalized with respect to the maximum force, the $\mathrm{P}-\mathrm{V}$ curves are also found to be identical in shape. Simultaneous recordings of the MgATPase activity and isometric force development showed that, despite $\sim$ twofold increase in $\mathrm{Ca}^{2+}$-activated force at low ionic strength $(50 \mathrm{mM})$, the MgATPase activity of the fibres did not change appreciably. These results strongly suggest that the enhancement of $\mathrm{Ca}^{2+}$-activated isometric force results from $\sim$ twofold increase of the force generated by individual myosin heads, but not from increase in the number of myosin heads responsible for force generation. This idea is supported by our recent finding using the technique of liquid cell electron microscopy that the amplitude of ATP-induced power stroke in individual myosin heads in hydrated myosin filaments increases from $\sim 3.3 \mathrm{~nm}$ to $>4 \mathrm{~nm}$ in the nearly isometric condition, in which gross myofilament sliding does not take place due to a small proportion of ATP-activated myosin heads [20]; since myosin heads perform power stroke by pulling adjacent elastic structures if gross myofilament sliding does not take place in the isometric condition, the larger the force generated by individual myosin heads, the larger is the amplitude of their power stroke pulling adjacent structures. 
As described above, $P-V$ relation is extremely useful in obtaining insights into the molecular mechanism, with which various factors affect contraction characteristics and MgATPase activity of skinned muscle fibers.

\section{Conclusion}

In this article, we presented an overview about research work on the P-V relation in whole muscles, single intact muscle fibers, and single skinned muscle fibers. Since hill's first description of the hyperbolic P-V relation, the Hill equation, $(P+a) V=b(P o-P)$, has been taken as evidence that muscle can regulate its energy output depending on the amount of load imposed on it. This fundamental property of contracting muscle or muscle fiber has been explained in terms of distribution of A-M link at both sides of the equilibrium position of myosin head based on assumptions of rate constants $f$ and $g$. Up to the present time, however, no contraction models can explain all the experimental results obtained from contracting muscle or muscle fibers. Muscle contraction is still filled with a number of mysteries. In our opinion, experiments on skinned muscle fibers are most important to reach full understanding on the molecular mechanism of muscle contraction, despite their inherent problems.

\section{Acknowledgements}

We wish to thank Drs. Teizo Tsuchiya, Takakazu Kobayashi and Hiroyuki Iwamoto for their contribution to the experiments described in this article. Our thanks are also due to Mr. Matthew Brokowski of Aurora Scientific Inc. for his kind help in the instrumentation of our research work currently in progress.

\section{References}

1. Hill AV (1938) The heat of shortening and the dynamic constants of muscle. Proc Roy Soc B 126: 136-195.

2. Podolsky RJ (1961) The nature of the contractile mechanism in muscle. In Biophysics of Physiological and Pharmacological Actions. Am Ass Adv 1Sci 461-482.

3. Huxley HE, Hanson J (1954) Changes in the cross-striations of muscle during contraction and stretch and their structural interpretation. Nature 175: 973-976.

4. Huxley AF (1957) Muscle structure and theories of contraction. Prog Biophys Bophys Chem 7: 255-318.

5. Edman KAP (1988) Double-hyperbolic force-velocity relation in frog muscle fibres. J Physiol 494: 301-321.
6. Iwamoto H, Sugaya R, Sugi H (1990) Force-velocity relation of frog skeletal muscle fibres shortening under constantly increasing load. J Physiol 422: 185-202.

7. Cecchi G, Colomo F, Lombardi V (1978) Force-velocity relation in normal and nitrate-treated frog single muscle fibres during rise of tension in an isometric tetanus. J Physiol 285: 257-273.

8. Jewell BR, Wilkie DR (1958) An analysis of the mechanical components in frog's striated muscle. J Physiol 143: 515-540.

9. Civan MM, Podolsky RJ (1966) Contraction kinetics of striated muscle fibres following quick changes in load. J Physiol 184: 511-534.

10. Podolsky RJ, Nolan (1973) Muscle contraction transients, cross-bridge kinetics and the Fenn effect. Cold Spring Harbor Symp Quant Biol 37: 661-668.

11. Sugi H, Tsuchiya $T$ (1981) Isotonic velocity transients in frog muscle fibres following quick changes in load. J Physiol 319: 239-252.

12. Brenner B (1998) Muscle mechanics II: skinned muscle fibres. In Current Methods in Muscle Physiology, Oxford University Press, Oxford 33-69.

13. Woledge RC, Curtin NA, Homsher E (1985) Energetic Aspects of Muscle Contraction. Academic Press, London.

14. Stienen GJM (2016) ATP utilization in skeletal and cardiac muscle: economy and efficiency. In Muscle Contraction and Cell Motility: Fundamentals and Developments. Pan Stanford Publishing, Singapore, 245-267.

15. Yamada T, Abe O, Kobayashi T, Sugi H (1993) Myofilament sliding per ATP molecule in rabbit muscle fibres studied using laser flash photolysis of caged ATP. J Physiol 466: 229-243.

16. Sugi H, Kobayashi T, Gross T, Noguchi K, Karr T, et al. (1992) Contraction characteristics and ATPase activity of skinned muscle fibers in the presence of antibody to myosin subfragment-2. Proc Natl Acad Sci USA 89: 6134-6137.

17. Thames MD, Teichholz LE, Podolsky RJ (1974) lonic strength and the contraction kinetics of skinned muscle fibers. J Gen Physiol 63: 509-530.

18. Gulati J, Podolsky RJ (1981) Isotonic contraction of skinned muscle fibers on a slow time base. J Gen Physiol 78: 233-257.

19. Sugi H, Abe T, Kobayashi T, Chaen S, Ohnuki Y, et al. (2013) Enhancement of force generated by individual myosin head in skinned rabbit psoas muscle fibers at low ionic strength. PLOS ONE 8: e63658.

20. Sugi H, Chaen S, Akimoto T, Minoda H, Miyakawa T, et al. (2015) Electron microscopic recording of myosin head power stroke in hydrated myosin filaments. Sci Rep 5: 15700. 